\title{
Premières mentions et répartition de la Salamandre sombre du Nord, Desmognathus fuscus, sur la rive nord du fleuve Saint-Laurent, au Québec
}

\author{
JeAn-François DesRoches ${ }^{1}$ et DANiEl Pouliot $^{2}$ \\ ${ }^{1}$ Collège de Sherbrooke, Département des Techniques d'écologie appliquée, 475 du Parc, Sherbrooke, Québec J1K 4K1 Canada \\ ${ }^{2}$ Laboratoire d'écologie des eaux douces, Université du Québec à Trois-Rivières, 3351 Boulevard des Forges, C.P. 500, Trois- \\ Rivières, Québec G9A 5H7 Canada
}

Desroches, J.-F., et D. Pouliot. 2005. Premières mentions et répartition de la Salamandre sombre du Nord, Desmognathus fuscus, sur la rive nord du fleuve Saint-Laurent, au Québec. Canadian Field-Naturalist 119(1): 105-109.

La Salamandre sombre du Nord (Desmognathus fuscus) est une espèce qui a fait l'objet de plusieurs inventaires au Québec étant donné qu'elle y est à la limite nord de sa répartition. Nous rapportons les premières observations de cette espèce au nord du fleuve Saint-Laurent, depuis Trois-Rivières, à l'ouest, jusqu'à Cap-Tourmente, vers l'est. Ces mentions ont été faites de 1987 à 2003 , dans 20 sites. La majorité des sites où fut découverte la Salamandre sombre du Nord sont des ruisseaux et des résurgences d'eau.

Mots-Clés: Desmognathus fuscus, Salamandre sombre du Nord, aire de distribution, répartition, habitat, Québec.

The Northern Dusky Salamander (Desmognathus fuscus) has been searched for extensively at its presumed northern range limit in Québec, south of the St-Lawrence River. We report the first records of this species north of the St-Lawrence River, from Trois-Rivières, in the west, to Cap-Tourmente in the east. These observations were made between 1987 and 2003 , at 20 sites. Most of the sites where the Northern Dusky Salamander was found are streams and seepages.

Key Words: Desmognathus fuscus, Northern Dusky Salamander, distribution, habitat, Québec.

La Salamandre sombre du Nord (Desmognathus fuscus) se retrouve à la limite nordique de sa répartition dans le sud de la province de Québec (Cook 1984). Les mentions historiques les plus nordiques de l'espèce ont été faites à Lac Trois-Saumons, comté de $1^{\prime}$ Islet $\left(47^{\circ} 08^{\prime} \mathrm{N}, 70^{\circ} 12^{\prime} \mathrm{O}\right)$ et à Saint-André, comté de Kamouraska $\left(47^{\circ} 38^{\prime} \mathrm{N}, 69^{\circ} 40^{\prime} \mathrm{O}\right)$, deux localités situées sur la rive sud du fleuve Saint-Laurent (Denman 1963). Bleakney (1958) avait émis l'hypothèse que le fleuve Saint-Laurent avait constitué une barrière à la dispersion de la Salamandre sombre du Nord. Bider et Matte (1991), dans la version détaillée de l'Atlas des amphibiens et des reptiles du Québec, mentionnent : «Puisque cette salamandre est activement recherchée par plusieurs herpétologistes, on peut donc penser que sa répartition décrite ici est assez complète». Or, en 1987, nous avons eu la surprise de la découvrir pour la première fois sur la rive nord du Saint-Laurent

En 1987, JFD trouva quelques spécimens de l'espèce à Boischatel, à l'est de Québec, dans des eaux de résurgence sur les rives abruptes de la rivière Montmorency. Ils ont été formellement identifiés par luimême en 1990, puis localisés approximativement dans l'Atlas des amphibiens et reptiles du Québec (Bider et Matte 1994). Des recherches visant à découvrir d'autres populations furent entreprises dès 1996 et 1998 par les auteurs. En 2001, une Salamandre sombre du Nord fut trouvée par DP à la rivière Jean-Larose, près du mont Sainte-Anne, à $28 \mathrm{~km}$ au nord-est des mentions faites à la rivière Montmorency. Cette découverte ouvrait la porte à la possibilité que cette salamandre puisse être plus répandue que prévu dans la région.
Un inventaire spécifique fut ainsi réalisé en 2002. Il avait comme objectif de documenter la répartition de l'espèce sur la rive nord du Saint-Laurent, et d'obtenir une idée générale de l'habitat de cette espèce à la limite nordique de sa répartition.

\section{Méthodologie}

L'inventaire de 2002 visant la découverte de la Salamandre sombre du Nord à d'autres endroits sur la rive nord du Saint-Laurent a été réalisé dans 40 nouveaux sites. Les sites ont été localisés préalablement sur des cartes topographiques 1: 50000 ou choisis au hasard sur le terrain. La méthodologie utilisée est similaire à celle de Weller (1977) et consiste en des recherches variant de 15 à 60 minutes dans les ruisseaux et sur les rives de cours d'eau, en soulevant les débris au sol. La présence et l'absence de la Salamandre sombre du Nord, le nombre d'individus, leur stade de développement, l'habitat, la date et la localisation au GPS en NAD83 ont été notés à chacun des sites visités (annexe I). Les sites situés à proximité, mais séparés par un secteur où l'habitat est moins propice, ont été considérés et cartographiés séparément malgré qu'ils puissent concerner le même cours d'eau. Au moins un spécimen a été récolté à la plupart des sites, sauf lorsque ceux-ci étaient situés à proximité l'un de l'autre. Les salamandres récoltées ont été déposées au Musée canadien de la nature sous les numéros CMNAR 35713-35729.

\section{Résultats et discussion}

De 1987 à 2003, la présence de la Salamandre sombre du Nord a pu être confirmée dans 20 sites situés 
sur la rive nord du Saint-Laurent. Cinq d'entre eux ont été découverts de 1987 à 2001, 13 durant l'inventaire spécifique de 2002, et deux durant des recherches supplémentaires effectuées en 2003 plus à l'ouest. Ces 20 sites s'étalent d'ouest en est de la rivière Saint-Maurice, à Trois-Rivières, jusqu'à Cap-Tourmente (figure 1). Les sites situés aux extrémités (ouest et est) sont séparés par $160 \mathrm{~km}$. Toutes les observations ont été faites dans les contreforts du Bouclier Canadien, dans le domaine de l'Érablière à Tilleul, où l'altitude moyenne varie de 55 à $116 \mathrm{~m}$ (Robitaille et Saucier 1998). Au sein de cette aire, la Salamandre sombre du Nord semble en général localisée à des secteurs où le microhabitat est propice: eaux de suintement, zones vaseuses avec mousses par endroits et bordure des ruisseaux. Le long des grandes rivières où on la retrouve, comme les rivières Saint-Maurice, Batiscan et Montmorency, elle fut retrouvée dans des eaux de ruissellement localisées et habituellement situées à quelque distance de la rivière (de quelques mètres à plus de $300 \mathrm{~m}$ ), et non de façon régulière sur les rives des cours d'eau.

Les habitats dans lesquels fut trouvée la Salamandre sombre du Nord sont : les ruisseaux $(<3 \mathrm{~m}$ de largeur) $(59,0 \%$ des sites), les eaux de résurgence $(36,4 \%)$, et un fossé forestier sans eau $(4,6 \%)$. Ces habitats concordent avec ceux décrits dans la littérature : habitats forestiers aquatiques ou semi aquatiques, souvent abondante aux endroits où se retrouvent des résurgences d'eau et le long des petits ruisseaux forestiers bordés de roches ou autres abris (Bishop 1941; Petranka 1998).

La plus forte proportion de ruisseaux peut représenter un biais. En effet, l'espèce semble assez typique des eaux de résurgence, mais ces milieux sont difficilement repérables sur des cartes à l'échelle 1: 50 000, comparativement aux cours d'eau permanents qui sont bien visibles.

Au Canada, la Salamandre sombre du Nord est une espèce associée aux Appalaches (Bleakney 1958). Nos résultats démontrent cependant qu'elle semble bien établie sur la rive nord du Saint-Laurent, dans les contreforts du Bouclier Canadien. Comment l'espèce s'y est-elle rendue? À quel endroit a-t-elle traversé le fleuve Saint-Laurent? La topographie du territoire porte à croire que ce fut dans le secteur de la ville de Québec, et vraisemblablement à partir de l'embouchure de la rivière Chaudière (rive sud du SaintLaurent, en bordure du pont de la route 73) qu'elle aurait franchi le fleuve, tel que proposé par Bider et Matte (1994). La Salamandre sombre du Nord se retrouve dans la rivière Chaudière et a pu traverser le fleuve à cet endroit, où la largeur de ce dernier est plus étroite (moins de $1 \mathrm{~km}$ ) qu'aux autres secteurs (2 km et plus). De plus, à cet endroit, les Appalaches se rendent à proximité du Saint-Laurent, ce qui n'est pas le cas plus à l'ouest où une large bande de la vallée du Saint-Laurent sépare les Appalaches du fleuve.
Suite à son arrivée sur la rive nord, la Salamandre sombre du Nord se serait ensuite répandue vers l'ouest, dans le Bouclier Canadien, pour se répandre jusque dans la région de Trois-Rivières, quelques $115 \mathrm{~km}$ plus à l'ouest. Vers l'est, il semble que sa répartition s'arrête aux environs du Cap-Tourmente, où l'altitude devient plus importante et la topographie plus accidentée. La végétation y change également, passant du domaine de l'Érablière à Tilleul à celui de la Sapinière à Bouleau jaune (Robitaille et Saucier 1998). D'ailleurs, ce secteur constitue la limite de répartition nord-est de plusieurs espèces végétales et animales, dont certains amphibiens et reptiles (Cook 1984). On ignore si la Salamandre sombre du Nord s'est répandue vers le nord mais les recherches effectuées ces dernières années n'ont pas permis de la repérer. Le secteur situé à l'ouest de Trois-Rivières, situé lui aussi dans le domaine de l'Érablière à Tilleul, semble particulièrement propice, mais à cet endroit la vallée du Saint-Laurent est présente sur une bande plus large et le Bouclier Canadien se retrouve donc plus au nord. Nos recherches effectuées plus à l'ouest dans le Bouclier Canadien, dans les Laurentides au nord de Montréal et en Outaouais, n'ont pas permis de trouver de Salamandres sombres du Nord mais seulement la Salamandre à deux lignes (Eurycea bislineata).

\section{Perspectives}

La découverte de la Salamandre sombre du Nord sur la rive nord du Saint-Laurent ouvre la porte à la possibilité que certaines espèces d'amphibiens, notamment les salamandres, soient répandues plus largement que l'on croit au Québec, et notamment sur la rive nord. En effet, la présence de cette espèce sur la rive nord du Saint-Laurent était ignorée jusqu'en 1990 (année où les premiers spécimens furent identifiés), malgré le fait que les salamandres de ruisseaux (la Salamandre sombre du Nord, la Salamandre à deux lignes et la Salamandre pourpre (Gyrinophilus porphyriticus) aient fait l'objet de quelques inventaires professionnels au Québec (Bider et Matte 1991; Pendlebury 1973; Bleakney 1958). Il apparaît possible que la Salamandre sombre du Nord soit encore plus répandue sur la rive nord du Saint-Laurent, de même que sur la rive sud vers l'est. Des inventaires spécifiques réalisés dans le futur pourraient permettre de tester cette hypothèse.

\section{Remerciements}

Les auteurs remercient tous ceux qui ont participé aux travaux de terrain : Daniel Banville, Josiane Bergeron, Joël Bonin, Nathalie Côté, Benoit Couture, Frédérick Desroches, Sébastien Desroches, Ian-Erik Gosselin, Isabelle Picard, Martin Savard et Vicky Tremblay. Ils remercient également Frederick W. Schueler, pour ses commentaires sur la version préliminaire du texte, de même que Mario Darsigny pour la réalisation de la carte de l'aire d'étude et de la localisation des sites. 


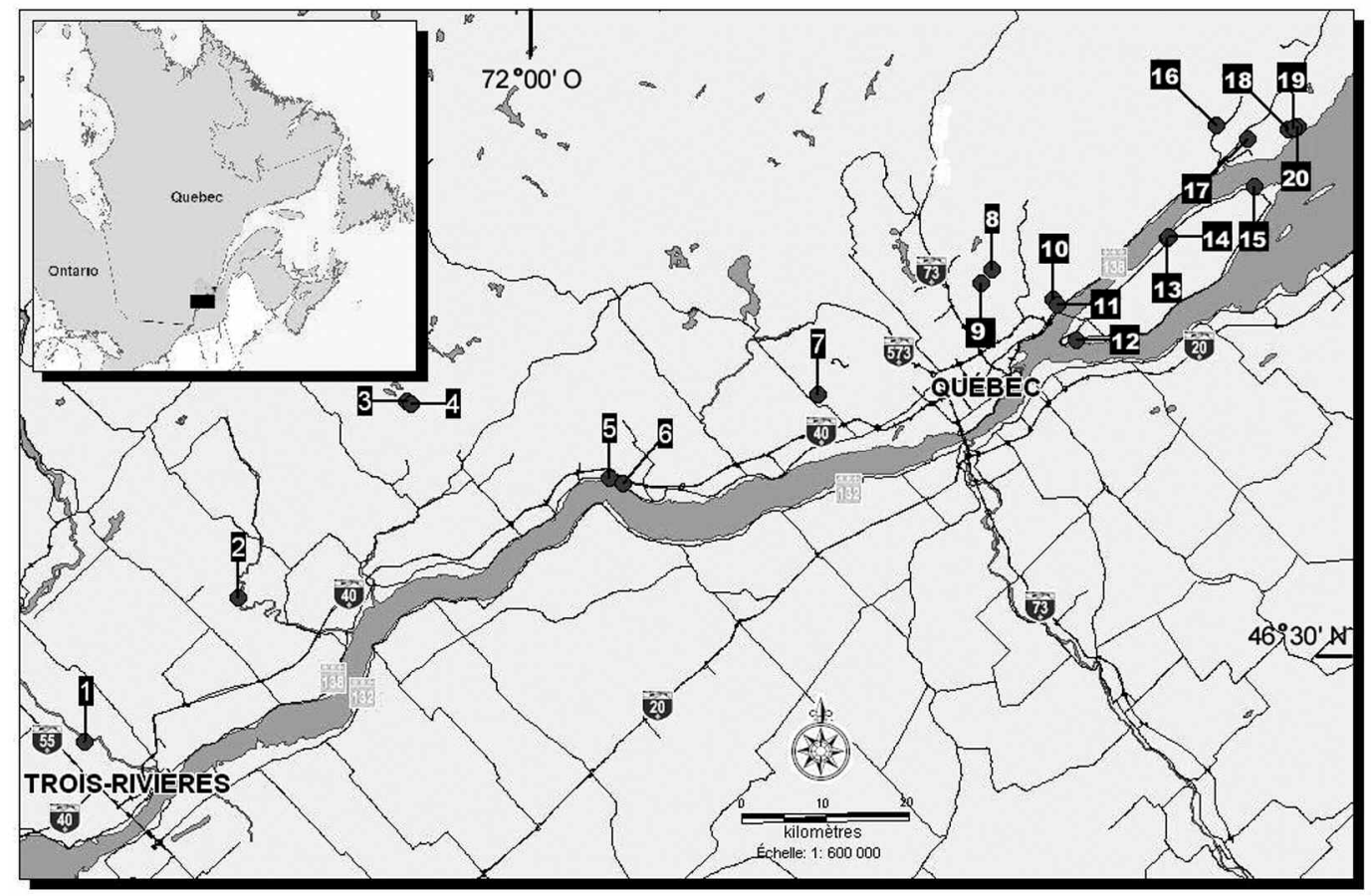

FiguRE 1. Localisation de l'aire d'étude au Québec (en médaillon) et des 20 sites où fut trouvée la Salamandre sombre du Nord sur la rive nord du Saint-Laurent, de 1987 à 2003.

1. Trois-Rivières, MRC de Francheville. $46^{\circ} 23^{\prime} 35^{\prime \prime} \mathrm{N} ; 7^{\circ} 38^{\prime} 58^{\prime \prime} \mathrm{O}$.

2 Saint-Narcisse, MRC de Francheville. 46 $33^{\prime} 14^{\prime \prime} \mathrm{N} ; 7^{\circ} 24^{\prime} 59^{\prime \prime} \mathrm{O}$.

3 Municipalité de paroisse de Saint-Alban, MRC de Portneuf. 46²46'14"N; $72^{\circ} 09^{\prime} 50^{\prime \prime} \mathrm{O}$.

4. Municipalité de paroisse de Saint-Alban, MRC de Portneuf. 46 $46^{\prime} 04^{\prime \prime N} ; 72^{\circ} 09^{\prime} 25^{\prime \prime} O$.

5. Municipalité de Cap-Santé, MRC de Portneuf. 46 $41^{\prime} 36^{\prime \prime N} ; 71^{\circ} 50^{\prime} 33^{\prime \prime O}$.

6. Municipalité de Cap-Santé, MRC de Portneuf. 46²41'17"N; $71^{\circ} 49^{\prime} 18^{\prime \prime O}$.

7. Municipalité de paroisse de Saint-Augustin-de-Desmaures, MRC de la Communauté Urbaine de Québec. $46^{\circ} 47^{\prime} 19^{\prime \prime} \mathrm{N} ; 71^{\circ} 31^{\prime} 08^{\prime \prime} \mathrm{O}$.

8. Charlesbourg, MRC de la Communauté Urbaine de Québec. 4655'35"N; 71¹4'54"O.

9. Charlesbourg, MRC de la Communauté Urbaine de Québec. 46 $54^{\circ} 44^{\prime \prime} \mathrm{N} ; 7^{\circ} 16^{\prime} 00^{\prime \prime} \mathrm{O}$.

10. Boischatel, MRC de la Côte-de-Beaupré. 46 $53^{\prime} 45^{\prime \prime} \mathrm{N} ; 71^{\circ} 09^{\prime} 07^{\prime \prime} \mathrm{O}$.

11. Boischatel, MRC de la Côte-de-Beaupré. $46^{\circ} 53^{\prime} 25^{\prime \prime} \mathrm{N} ; 71^{\circ} 08^{\prime} 47^{\prime \prime} \mathrm{O}$.

12. Sainte-Pétronille, MRC de l'Île-d'Orléans. 46 $51^{\prime} 10^{\prime \prime N} ; 71^{\circ} 06^{\prime} 55^{\prime \prime O}$.

13. Sainte-Famille, MRC de l'Île-d'Orléans. 46057'49"N; $70^{\circ} 58^{\prime} 24^{\prime \prime O}$.

14. Sainte-Famille, MRC de l'Île-d'Orléans. 46057'54"N; $70^{\circ} 58^{\prime} 17^{\prime \prime O}$.

15. Saint-François, MRC de l'île-d'Orléans. 4701'12"; $70^{\circ} 50^{\prime} 16^{\prime \prime} \mathrm{O}$.

16. Mont-Sainte-Anne, MRC de la-Côte-de-Beaupré. $47^{\circ} 05^{\prime} 04^{\prime \prime N} ; 70^{\circ} 54^{\prime} 01^{\prime \prime O}$.

17. Saint-Joachim, MRC de la-Côte-de-Beaupré. 4704'13"N; 7051'03"O.

18. Cap-Tourmente, MRC de la-Côte-de-Beaupré. 4704'55"N; $70^{\circ} 47^{\prime} 09^{\prime \prime} \mathrm{O}$.

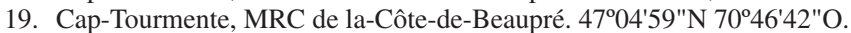

20. Cap-Tourmente, MRC de la-Côte-de-Beaupré. 4705'05"N; 7046'16"O.

Lesroutes provincials $20,40,55,73$, et 573 sont inclues.

\section{Littérature citée}

Bider, J.-R., et S. Matte. (compilé par). 1991. Atlas des amphibiens et reptiles du Québec 1988-1989-1990, version détaillée. Société d'histoire naturelle de la vallée du SaintLaurent et ministère du Loisir, de la Chasse et de la Pêche du Québec. Québec. 429 pages.

Bider, J.-R., et S. Matte. 1994. Atlas des amphibiens et reptiles du Québec. Société d'histoire naturelle de la vallée du Saint-Laurent et ministère de l'Environnement et de la Faune du Québec. Québec. 106 pages.
Bishop, S. C. 1941. The salamanders of New York. New York State Museum Bulletin (324). Albany, New York. 365 pages.

Bleakney, J. S. 1958. A zoogeographical study of the amphibians and reptiles of Eastern Canada. National Museum of Canada, Bulletin 155. 119 pages.

Cook, F. R. 1984. Introduction aux amphibiens et reptiles du Canada. Musée national des sciences naturelles et Musées nationaux du Canada. Ottawa, Canada. 211 pages. 
Denman, N. S. 1963. A range extension of the Dusky Salamander in Quebec. Canadian Field-Naturalist 77 :62.

Pendlebury, G. B. 1973. Distribution of the Dusky Salamander Desmognathus fuscus fuscus (Caudata: Plethodontidae) in Quebec, with special reference to a population from St. Hilaire. Canadian Field-Naturalist 87: 131-136.
Petranka, J. W. 1998. Salamanders of the United States and Canada. Smithsonian Institution Press. Washington and London. 587 pages.

Robitaille, A., et J.-P. Saucier. 1998. Paysages régionaux du Québec méridional. Les Publications du Québec. 213 pages + carte.

Weller, W. F. 1977. Distribution of stream salamanders in southwestern Quebec. Canadian Field-Naturalist 91 : 299-303.

Received 16 February 2004

Accepted 17 February 2005

\section{Annexe I. Détails sur les localités et les salamandres observées}

Chaque donnée est présentée de la manière suivante : \# du site (voir la figure 1), nom de la municipalité, nom de la MRC (municipalité régionale de comté), localisation GPS en NAD 83, type d'habitat (eaux de résurgence, ruisseau) et présence ou non de Salamandre à deux lignes, date, nombre d'individus de Salamandres sombres du Nord observés et stade, nombre de spécimens récoltès, nom des observateurs, numéro de catalogue musée canadien de la nature (amphibiens et reptiles) CMNAR.

1. Les Vieilles-Forges, MRC de Francheville. 46²3'35"N; 72³8'58"O. Environ $100 \mathrm{~m}$ à l'ouest de la rivière SaintMaurice. Habitat : Eaux de résurgence.

20 juillet 2003 : 2 adultes (1 récolté), obs. : J.-F. Desroches, D. Pouliot, I. Picard et N. Côté. CMNAR 35278.

2. Saint-Narcisse, MRC de Francheville. 46033'14"N; 72²4'59"O. Parc de la rivière Batiscan, environ 300 m à l'ouest de la rivière Batiscan. Habitat : Eaux de résurgence.

20 mai 2003 : 1 subadulte, obs. : D. Pouliot et M. Savard.

24 mai 2003 : 4 adultes (1 récolté) et 2 subadultes. : D. Pouliot et N. Côté. CMNAR 35729.

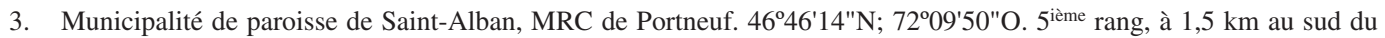
lac en Cœur. Habitat : Eaux de résurgence. Présence de Salamandres à deux lignes. 20 août $2002: 5$ adultes (1 récolté), JFD-02.10, obs. : D. Pouliot. CMNAR 35722.

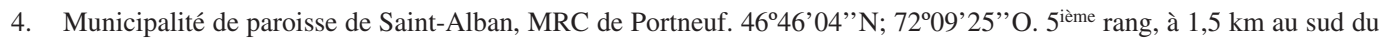
lac en Cœur. Habitat : ruisseau. 20 août $2002: 2$ adultes, obs. : D. Pouliot.

5. Municipalité de Cap-Santé, MRC de Portneuf. 46²41'36"N; 7150'33"O. 2,3 km à l'est de Portneuf, au nord de la route 138. Habitat : ruisseau. Présence de Salamandres à deux lignes. 29 juillet 2002 : 1 adulte (récolté) et 1 jeune, JFD-02.17, obs. : D. Pouliot. CMNAR 35725.

6. Municipalité de Cap-Santé, MRC de Portneuf. 4641'17"N; 7149'18"O. Ruisseau André-Jacques, 2,8 km au nordouest de Cap-Santé, entre l'autoroute 40 et la route 138. Habitat : ruisseau. Présence de Salamandres à deux lignes. 22 juillet $2002: 5$ adultes (1 récolté) et 1 jeune (récolté), JFD-02.19, obs. : D. Pouliot. CMNAR 35727.

7. Municipalité de paroisse de Saint-Augustin-de-Desmaures, MRC de la Communauté Urbaine de Québec. 4647'19"N; $71^{\circ} 31^{\prime} 08^{\prime \prime} O .1,3 \mathrm{~km}$ à l'est de la route 367 . Habitat : ruisseau. 9 août 2002 : 4 adultes (1 récolté), JFD-02.18, obs. : D. Pouliot. CMNAR 35726.

8. Charlesbourg, MRC de la Communauté Urbaine de Québec. 4655'35"N; 71¹4'54"O. Secteur du lac Des Roches. Habitat : fossé forestier.

31 mai 1998 : 1 adulte, obs. : J.-F. Desroches et F. Desroches.

9. Charlesbourg, MRC de la Communauté Urbaine de Québec. 46 $54^{\prime} 44^{\prime \prime N} ; 71^{\circ} 16^{\prime} 00^{\prime \prime O}$. Rivière Des Roches, en aval du lac Des Roches. Habitat : ruisseau. Présence de Salamandres à deux lignes.

17 mai $1996: 3$ adultes, obs. : J.-F. Desroches.

9 juillet 1997 : 1 subadulte, obs. : J.-F. Desroches.

31 juillet $2002: 1$ adulte (récolté) et 1 jeune, JFD-02.16, obs. : D. Pouliot. CMNAR 35724.

10. Boischatel, MRC de la Côte-de-Beaupré. 46 $53^{\prime} 45^{\prime \prime N} ; 7^{\circ} 09^{\prime} 07^{\prime \prime O}$. Rivière Montmorency en amont des chutes. Habitat : eaux de résurgence et ruisseau. Présence de Salamandres à deux lignes.

1987 : plusieurs trouvées; 2 adultes (JFD-70) et 1 larve (JFD-55) récoltés, obs. : J.-F. Desroches. CMNAR 35715, 35714. 23 avril $1990: 2$ adultes et quelques larves, obs. : J.-F.Desroches.

13 octobre $1990: 2$ adultes, obs. : J.-F. Desroches.

28 avril $1991: 2$ adultes, obs. : J.-F. Desroches.

30 mai $1991: 35$ adultes et jeunes +10 larves, obs. : J.-F. Desroches.

17 avril 1992: 1 adulte et 1 larve, obs. : J.-F. Desroches.

9 février 1993 : 1 larve, obs. : J.-F. Desroches. 
20 mars 1994 : 1 larve, obs. : J.-F. Desroches et F. Desroches.

3 mai 1995 : 2 adultes, obs. : J.-F. Desroches.

14 mars $1996: 6$ adultes ( 3 récoltés) et 2 larves, JFD-385, obs. : J.-F. Desroches et B. Couture. CMNAR 35717.

8 mai $1996: 5$ adultes et 3 jeunes, obs. : J.-F. Desroches.

17 mai $1997: 5$ adultes et 3 larves, obs. : J.-F. Desroches.

12 septembre $1999: 2$ adultes et 2 jeunes, obs. : J.-F. Desroches, J. Bonin et I. Picard.

18 octobre $1999: 2$ adultes, 1 jeune et 1 larve, obs. : J.-F. Desroches.

28 août $2002: 4$ adultes et 1 jeune, obs. : D. Pouliot et D. Banville.

11. Boischatel, MRC de la Côte-de-Beaupré. 4653'25"N; $71^{\circ} 08^{\prime} 47^{\prime \prime} \mathrm{O}$. Rivière Montmorency au pied des chutes. Habitat : eaux de résurgence. Présence de Salamandres à deux lignes.

1990 : quelques trouvées, obs. : J.-F. Desroches.

8 juillet $1991: 32$ adultes et jeunes, obs. : J.-F. Desroches.

12 septembre $1999: 2$ adultes et 1 sub-adulte, obs. : J.-F. Desroches, J. Bonin et I. Picard.

18 octobre $1999: 2$ larves, obs. : J.-F. Desroches.

12. Sainte-Pétronille, MRC de l'Île-d'Orléans. 4651'10"N; 7106'55"O. Ruisseau Marie-Anne, secteur ouest de l'Île d'Orléans, $200 \mathrm{~m}$ au nord de la route 368. Habitat : ruisseau. Présence de Salamandres à deux lignes.

14 juin $2002: 5$ adultes et 2 jeunes, obs. : D. Pouliot, V. Tremblay, D. Banville et N. Côté.

13. Sainte-Famille, MRC de l'Île-d'Orléans. 4657'49"N; 7058'24"O. Secteur centre de l'Île-d'Orléans, 1,2 km au sud-ouest de Sainte-Famille, au nord de la route 368. Habitat : ruisseau. Présence de Salamandres à deux lignes. 14 juin $2002: 1$ adulte, obs. : D. Pouliot, V. Tremblay et N. Côté.

14 Sainte-Famille, MRC de l'île-d'Orléans. 4657'54"N; 7058'17"O. Habitat : ruisseau. Présence de Salamandres à deux lignes.

25 juillet 2002 : 1 adulte, JFD-02.15, obs. : D. Pouliot. CMNAR 35723.

15. Saint-François, MRC de l'Île-d'Orléans. 4701'12"; 7050'16"O. Ruisseau du Moulin, secteur est de 1'Île-d'Orléans, $800 \mathrm{~m}$ au nord de la route 368. Habitat : ruisseau. Présence de Salamandres à deux lignes.

17 juin $2002: 2$ adultes, obs. : D. Pouliot et N. Côté.

16. Mont-Sainte-Anne, MRC de la-Côte-de-Beaupré. $47^{\circ} 05^{\prime} 04^{\prime \prime N}$; $70^{\circ} 54^{\prime} 01^{\prime \prime O}$. Rivière Jean-Larose, $840 \mathrm{~m}$ au nord de la route 360. Habitat : eaux de résurgence. Présence de Salamandres à deux lignes.

15 août $2001: 1$ adulte (récolté), JFD-01.10, obs. : D. Pouliot et I.-E. Gosselin. CMNAR 35718.

10 juin 2002 : 1 adulte (photographié), obs. : D. Pouliot, V. Tremblay et D. Banville.

17. Saint-Joachim, MRC de la-Côte-de-Beaupré. 4704'13"N; $70^{\circ} 51^{\prime} 03^{\prime \prime} O$. Ruisseau des Carrières, en bordure est de $1^{\prime}$ avenue Royale. Habitat : eaux de résurgence. Présence de Salamandres à deux lignes dans ruisseau à proximité.

4 juillet 2002 : 1 adulte, JFD-580, obs. : J.-F. Desroches et V. Tremblay. CMNAR 35719.

18. Cap-Tourmente, MRC de la-Côte-de-Beaupré. 4704'55"N; 7047'09"O. Ruisseau de la Friponne. Habitat : eaux de résurgence et ruisseau. Présence de Salamandres à deux lignes.

28 août 2002 : 2 adultes (1 récolté), JFDA-02.9, obs. : D. Pouliot, D. Banville et V. Tremblay. CMNAR 35721.

19. Cap-Tourmente, MRC de la-Côte-de-Beaupré. 4704'59"N 7046'42"O. Ruisseau Pierré. Habitat : ruisseau. Présence de Salamandres à deux lignes.

28 août $2002: 2$ adultes, obs. : D. Pouliot, D. Banville et V. Tremblay.

20. Cap-Tourmente, MRC de la-Côte-de-Beaupré. 4705'05"N; 7046'16"O. Ruisseau Pierré. Habitat : ruisseau. Présence de Salamandres à deux lignes.

28 août 2002 : 2 adultes (1 récolté), JFD-02.8, obs. : D. Pouliot, D. Banville et V. Tremblay. CMNAR 35720. 